EP-208

\title{
Granular cell tumor of abdominal wall
}

\author{
Aekta NEHA', Amar RANJAN ${ }^{* 2}$ \\ 'Department of Pathology, Patna Medical College, Patna, Bihar, India \\ ${ }^{2}$ Department of Laboratory Oncology, DR. BRA Institute-Rotary Cancer Hospital, \\ All India Institute of Medical Sciences, New Delhi, India
}

Introduction: Granular Cell Tumor (GrCT) is a rare benign tumor, likely arising from Schwann cells and accounts for $0.5 \%$ of soft tissue tumor. About $0.5 \%$ to $2 \%$ cases are malignant.

Methods: The case was retrieved from the hospital record.

Results: A 46 year old male presented with a painful nodular mass in flank for six months. It was firm, immobile subcutaneous swelling, fixed to skin with irregular margin measuring $3 \times 2 \mathrm{~cm}$. Ultrasonography revealed it as localized lesion. FNAC was inconclusive. Excision biopsy showed large polygonal cells with granular eosinophilic cytoplasm and eccentric nucleus with entrapped adnexal structure invading deep into adipose tissue. Cells were PAS positive \& Diastase resistance. On immunohistochemistry, cells were positive for S-100 and CD-68 and negative for SMA \& Vimentin. Diagnosis of GrCT of benign nature was made. Postoperative course was uneventful after 1.5 years of follow-up.

Conclusions: GrCT was originally named as myoblastoma, but currently, it is considered to be neural in origin. It is commonly found in females of 2 nd to 4 th decade, most common site being tongue, oral cavity. Fanburg Smith Criteria distinguishes benign from malignant lesions, that includes (i) necrosis (ii) spindling (iii) vesicular nuclei with large nucleoli (iv) increased mitotic activity (> 2 mitosis/ HPF) (v) increased nucleo-cytoplasmic ratio (vi) pleomorphism. If out of these $6 ;>3$ features are indicates malignancy. Abdominal wall GrCT is rare. We found only 11 cases of abdominal wall GCT in medical literature. Prognosis is good in non-malignant cases. Excision is the treatment choice, rarely recurs. 